\title{
Observation of the Forbush decrease of 22 June 2015 with the LAGO detector in Brazil
}

\author{
Anderson Campos Fauth*1, Henrique Vieira de Souza1, for the LAGO Collaboration ${ }^{2}$ \\ ${ }^{1}$ Instituto de Física "Gleb Wataghin", Universidade Estadual de Campinas \\ Rua Sérgio Buarque de Holanda 777 CEP 130830859 Campinas-SP,Brazil \\ 2 The Latin American Giant Observatory (LAGO), http://lagoproject.org/ \\ See full list of members and institutions at http://lagoproject.org/collab.html \\ E-mail: fautheifi.unicamp.br
}

\begin{abstract}
The Latin American Giant Observatory (LAGO) is a widespread cosmic ray observatory which uses water-Cherenkov Detectors (WCD) deployed in nine Latin American countries, being able to detect transient events or long term modulation effects simultaneously at places having different geomagnetic rigidity cut-offs and altitudes. In this work, results of studies on cosmic ray muon flux variations during the Forbush decrease of 22 June 2015 registered by the LAGO detector installed at the University of Campinas, Brazil, $22^{\circ} 49^{\prime} 03^{\prime \prime} S, 47^{\circ} 04^{\prime} 11^{\prime \prime} W$ at $650 \mathrm{~m}$ a.s.l., the TANCA detector, are presented. TANCA is a cylindrical polyethylene tank of $10 \mathrm{~m}^{2}$ containing a sealed liner with a reflective inner surface. The liner contains 11400 liters of ultra-pure water. Cherenkov light produced by the passage of particles through the water is collected by three nine-inch-diameter photomultiplier tubes. The experimental setup and detector performance is also presented. The studies on Forbush decrease contain comparisons with neutron monitors and indices of Earth's magnetic activity.
\end{abstract}

35th International Cosmic Ray Conference - ICRC2017

10-20 July, 2017

Bexco, Busan, Korea

${ }^{*}$ Speaker. 


\section{Introduction}

The Latin American Giant Observatory (LAGO)[1] is a collaborative and non-centralized network of institutions from ten countries (Argentina, Bolivia, Brazil, Colombia, Ecuador, Guatemala, Mexico, Peru, Venezuela and Spain) which maintain an extended cosmic ray observatory composed of water-Cherenkov Detectors (WCD) placed throughout Latin America. Every LAGO site consists of a single WCD or a small array, they are located at different altitudes and cover a large range of geomagnetic rigidity cut-offs. This collaboration, among other astrophysical researches, is studying short-term modulations associated with interplanetary transient perturbations, that can cause a phenomena known as a Forbush Decrease. Water-Cherenkov Detectors placed on the ground detect mainly muons produced primarily by galactic cosmic rays. The LAGO detectors permit one to study space weather through the solar modulation of galactic cosmic rays at energies that neutron monitors can't reach. Forbush Decrease is one of the most important solar events that disturb the terrestrial magnetic field. They are generally associated with a Coronal Mass Ejection (CME) that can causes magnetic storms. If large CMEs are directed towards Earth, a great space storm can be developed and disable satellites, increase radiation exposure for airline passengers, black out radio and Global Positioning System communications. In the worst case, geomagnetic storms can disrupt electric power distribution in low latitude cities. The study of Forbush Decrease with water-Cherenkov Detectors can be useful to understand Forbush Decrease precursor signatures in the muon flux some hours before the magnetic storm arrives on Earth. It can be used to study the characteristics of magnetic field compression associated with the CME too.

\section{The TANCA Detector}

TANCA is a nickname for the TAnk detector of CAmpinas. It is one WCD of the LAGO observatory and is placed at the campus of the University of Campinas (UNICAMP), in southeastern Brazil. The tank (see Figure 1(a)) is a cylindrical polyethylene tank of $10 \mathrm{~m}^{2}$ containing a sealed liner with a Tyvec $R$ reflective inner surface. The liner contains 11400 liters of ultra pure water and the Cherenkov light produced by the passage of relativistic particles through the water is collected

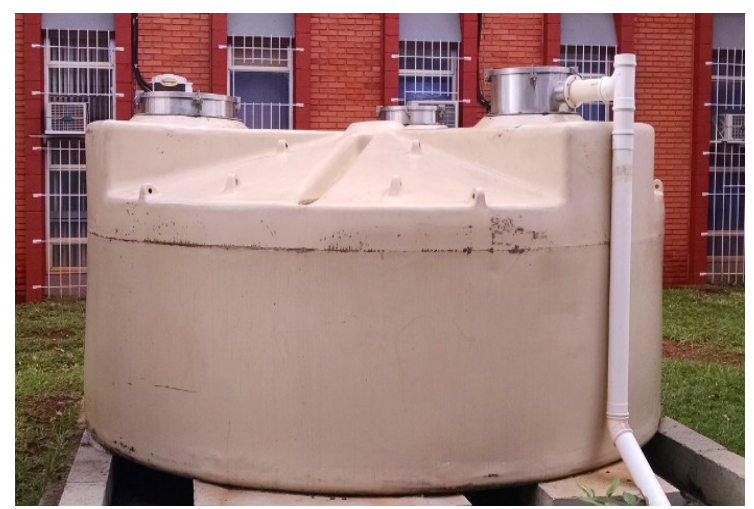

(a) Water-Cherenkov Detector TANCA

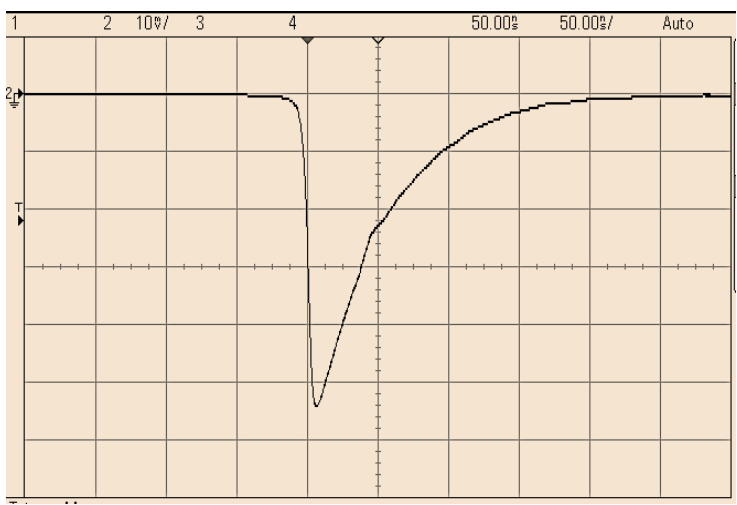

(b) Pulse of TANCA generated by a single muon. Scale: $50 \mathrm{~ns} ; 10 \mathrm{mV}$ per division.

Figure 1: The water-Cherenkov Detector TANCA and its single muon pulse. 


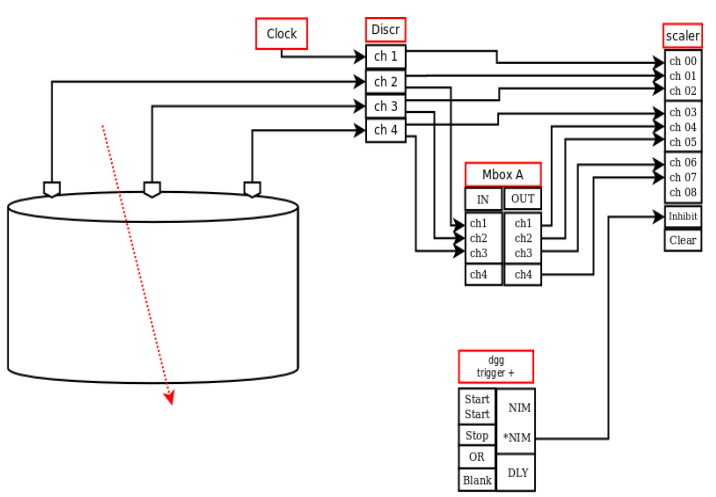

(a) Schematic diagram of the data aquisition system

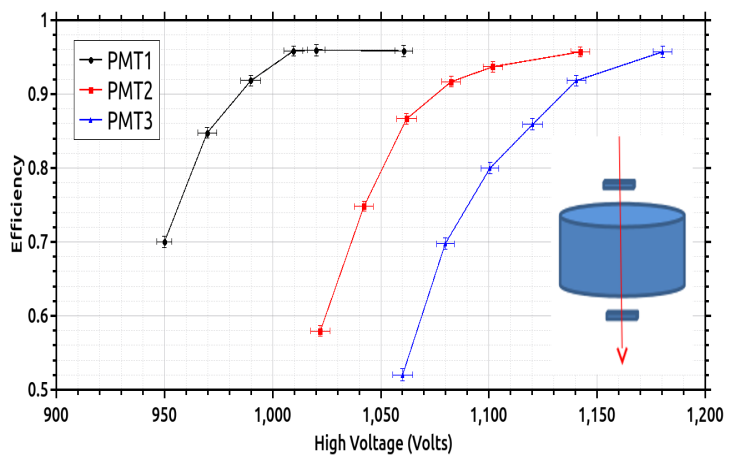

(b) Plateaux of the triple fold coincidece (see text for explanation).

Figure 2: Data acquisition schematic diagram and detector muon count plateau efficiency.

by three Photonis XP1805, nine-inch-diameter, photomultiplier tubes (PMT). Figure 1(b) shows a detector pulse produced by one muon crossing the tank.

\subsection{Experimental setup and detector calibration}

The flux of muons is registered with a CAMAC system containing one LeCroy Programmable Dual Gate Generator, model 2323A, a CAEN programmable logic unit, model C85, a LeCroy Scaler of $100 \mathrm{MHz}$, model 2551 and a CAEN eight-fold fast discriminator, model N96. A Hewlett Packard pulse generator, model 8012B, is used as a constant clock to verify sporadic electromagnetic noise. A schematic diagram of the data acquisition system is shown in Figure 2(a). Every second the following information is registered: time stamp; clockHP; counts for single coincidence $(\operatorname{ch} 1, \operatorname{ch} 2, \operatorname{ch} 3)$; double coincidence $(\operatorname{ch} 1+\operatorname{ch} 2, \operatorname{ch} 1+\operatorname{ch} 3, \operatorname{ch} 2+\operatorname{ch} 3)$ and triple coincidence $(\operatorname{ch} 1+\operatorname{ch} 2+\operatorname{ch} 3)$. The accuracy of the count interval is $0.2 \%$, defined by the LeCroy Programmable Dual Gate Generator. Every ten minutes data of atmospheric condition are registered (barometric pressure, temperature of air, humidity, rain, rainfall, wind speed and direction), the information is obtained from the University of Campinas weather station. The count efficiency is measured using two small, 40x38x2.5 $\mathrm{cm}^{3}$, plastic scintillators, called rackets, to select vertical muons. The detector count efficiency for muons is calculated as the ratio of three-fold coincidence (rackets and detector) by the rackets two-fold coincidence, after subtracting the spurious signals. The graph of the count efficiency versus high voltage is shown in Figure 2(b). In the figure an outline of the geometrical arrangement used for the efficiency measurement is also shown at the bottom right corner. The TANCA muon count efficiency is $(95.7 \pm 0.8) \%$.

\section{Forbush Decrease of June 2015}

The flux of muons depends on the barometric pressure and this correlation can be approximated with an exponential behavior, $I=I_{0} \exp \left[-\beta\left(P-P_{0}\right)\right]$, where $\beta$ is the barometric coefficient. The TANCA barometric coefficient is calculated having a value of $\beta=(0.108 \pm 0.006) \% /$ mbar. All Forbush analyses are done with data corrected by the barometric coefficient. 


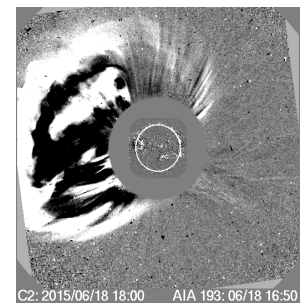

(a) CME1

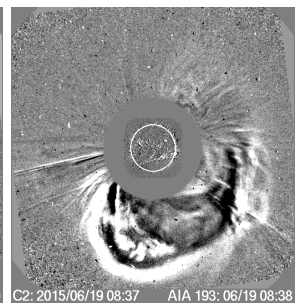

(b) CME2

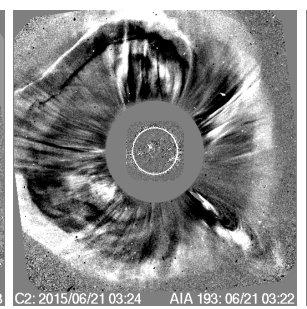

(c) CME3

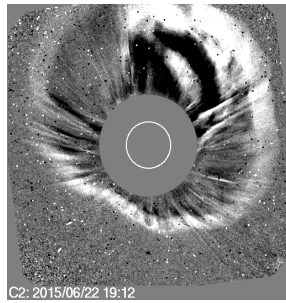

(d) CME4

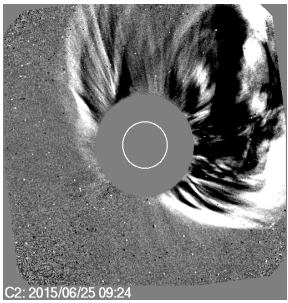

(e) CME5

Figure 3: CME pictures at ejection moment[2].

Five Coronal Mass Ejections were detected by TANCA in June 2015. The first CME arrived on $21^{\text {th }}$ June and the last on $27^{\text {th }}$. Figure 3 shows five pictures of CMEs, which arrived on Earth, registered by $\mathrm{C} 2$ chronograph of the SOHO/LASCO, at initial ejection moment on Sun [2]. The disturbance of the Earth's magnetosphere caused by these CMEs was detected by the LAGO waterCherenkov detector at UNICAMP.

In Figure 4(a) a comparison of TANCA measurements and the McMurdo neutron monitor(Ross Island, Antarctica, $77.9^{\circ} \mathrm{S}, 116.6^{\circ} \mathrm{E}$, altitude $48 \mathrm{~m}$ a.s.1.) [3] is shown. The dashed vertical lines shows the CME arrival time on Earth. Although the McMurdo signal has a lower geomagnetic cutoff since it is located at the South Pole, the TANCA detection shows CME precursors generated by the CME shock wave. The TANCA has higher statistics with about 1,600 $\mathrm{Hz}$ and 1,800 $\mathrm{Hz}$ cosmic ray counting for the triple coincidence and for the single counting respectively. The Pierre Auger Observatory (Malargue, Argentina, $69.3^{\circ} \mathrm{S}, 35.3^{\circ} \mathrm{W}$, altitude $1400 \mathrm{~m}$ a.s.l.) makes the fifteen minutes time averaged data of the "single particle technique", called Scaler Mode, publicly available [4]. In Figure 4(b) a comparison of the TANCA and the Scaler Mode Forbush Decrease detection is shown. Also in this case there is a clear agreement between the experiments, showing the TANCA more precise measurements since the CMEs precursors are shown more clearly. Finally in Figure 4(c) a comparative plot of TANCA and the Disturbance storm time (Dst)[5] index, showing the Earth's magnetic field is weakening, and also the TANCA measurement is shown.

\section{Conclusion}

The installation and calibration of the TANCA, a water-Cherenkov Detector of the LAGO Collaboration, is presented. The detector, sited at University of Campinas, Brazil, is inside the South Atlantic Magnetic Anomaly (SAA) acquiring date continuously, allowing measurements of the muon flux variations associated with solar events with high statistics and good temporal resolution.

The present study of the TANCA performance shows it has a muon count efficiency of $(95.7 \pm$ $0.8) \%$ and the data acquisition system is stable enough for research on transient signals caused by solar energetic events. In June 2015 five CMEs were ejected towards the Earth initiating a big Forbush event, result of those CMEs overlapping. A comparative analysis of this Forbush event with TANCA, McMurdo neutron monitor and Scaler Mode of the Pierre Auger Observatory shows complete concordance. This TANCA result shows the possibility of a future study of precursors of magnetic storms using muons detected by water-Cherenkov Detectors. TANCA is enabling the 


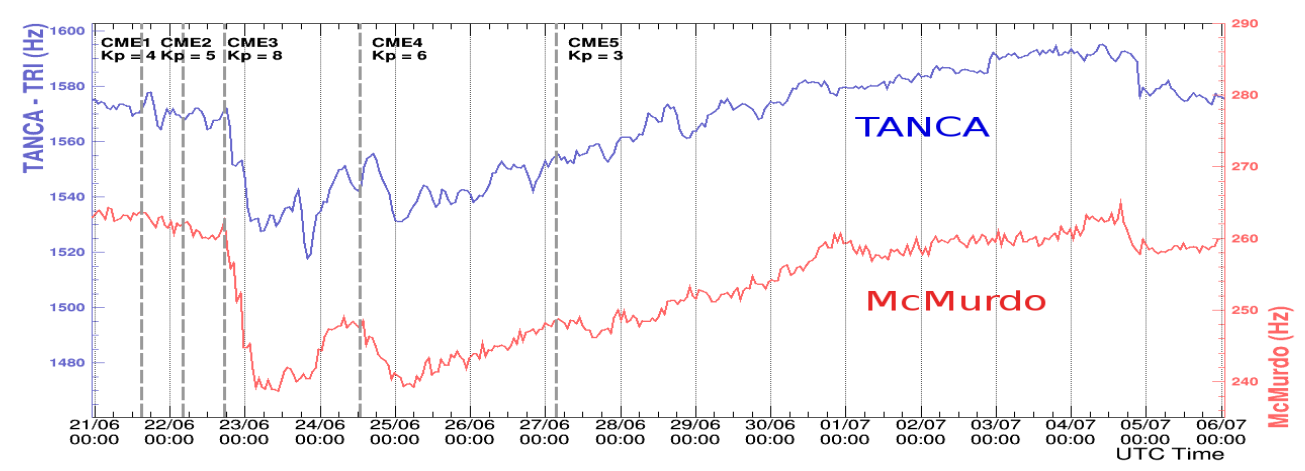

(a) TANCA and McMurdo neutron monitor detections.

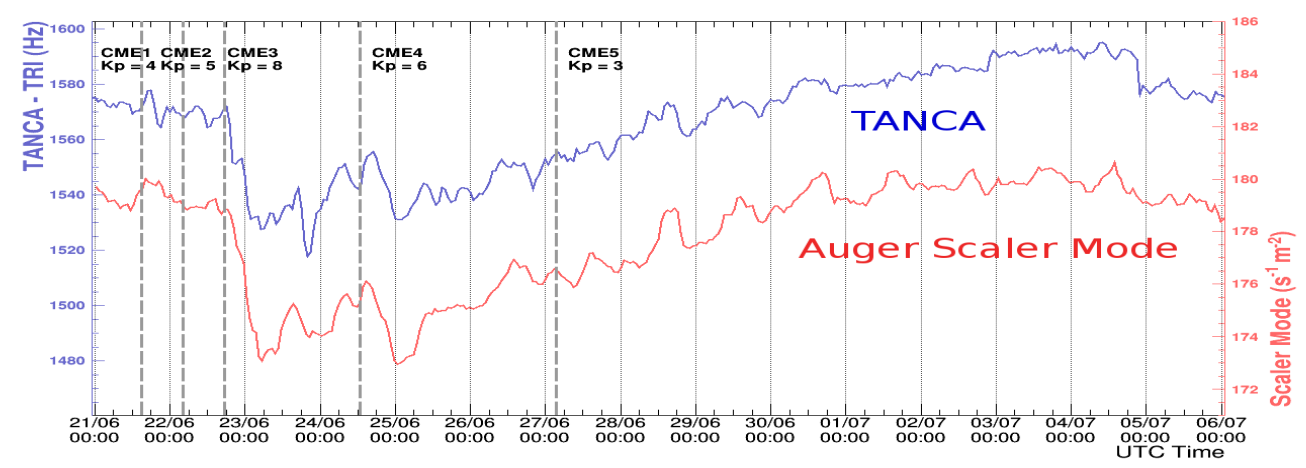

(b) TANCA and Pierre Auger Observatory Scaler Mode results.

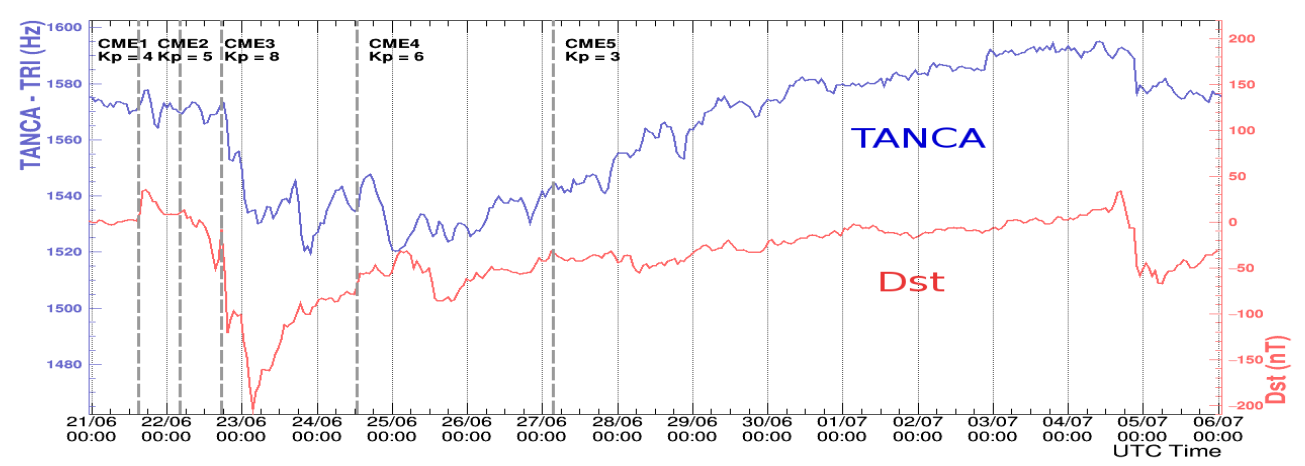

(c) TANCA and Disturb Storm Time (Dst) measurements.

Figure 4: Confrontation of TANCA Forbush detection with McMurdo,Auger Scaler Mode and Dst.

study of solar events at higher energies than space and neutron monitor experiments inside the SAA. The next step is to analyze solar events in conjunction with other LAGO detectors, deepening the Forbush analyses and increasing the regional integration of the Astrophysics community in Latin America. 


\section{Acknowledgments}

This work is supported by the São Paulo Research Foundation (FAPESP), grants 2017/106466, 2010/07359-6, 2011/50193-4), and The Brazilian National Council for Scientific and Technological Development (CNPq), grants 446335/2014-9, 309363/2013-6. We acknowledge the Pierre Auger Observatory and the University of Delaware (NSF grant ANT-0739620) for their open data policy making their data freely available through their web sites.

\section{References}

[1] H. Asorey, S. Dasso, The LAGO Collaboration, LAGO: the Latin American Giant Observatory, in proceedings of 34th International Cosmic Ray Conference, POS ( ICRC2015) 247.

[2] CDAW Data Center by NASA and The Catholic University of America in cooperation with the Naval Research Laboratory.https://cdaw.gsfc.nasa.gov/CME_list/, accessed 16/04/2017.

[3] The data from McMurdo were provided by the University of Delaware with support from the U.S. National Science Foundation under grant ANT-0739620. http://neutronm.bartol.udel.edu/, accessed $16 / 04 / 2017$

[4] The Pierre Auger Collaboration, The Pierre Auger Observatory Scaler Mode for the Study of Solar Activity Modulation of Galactic Cosmic Rays, Journal of Instrumentation, 6, P01003 (2011).

[5] Disturbance storm time (Dst) index,http://wdc.kugi.kyoto-u.ac.jp/dstdir/index.html, accessed 16/04/2017. 